Journal of

Accident and Emergency Medicine 1994 11, 243-245

\title{
A critical look at accident and emergency review clinics
}

\author{
A.K.MAITRA \& M.Q.CHOYCE
}

Accident and Emergency Department, Royal Victoria Infirmary, Newcastle upon Tyne

\section{SUMMARY}

The reasons for and appropriateness of referral of patients after initial visit to accident \& emergency (A\&E) follow-up clinics by the A\&E doctors were prospectively analysed in 500 patients. Fifteen per cent of patients were judged to have been inappropriately referred. Two main reasons for referral were the need for further treatment and uncertainty about diagnosis and management. The implications of reductions of A\&E clinic attendances are discussed.

Key words: accident and emergency, clinic, referral, review

\section{INTRODUCTION}

Attendances at A\&E departments in England have risen steadily from 9 million in 1980 to over 11 million in 1990/91. The percentage of those patients returning for review has dropped from $42 \%$ in 1980 to $22 \%$ in 1991 , representing a considerable saving of staff time and resources. ${ }^{1}$ This fall can be related to the development of the career and training structure within the specialty of A\&E medicine which has led to the appointment of dedicated A\&E consultants and senior registrars (and registrars) who provide regular on-site supervision, training and teaching of junior staff and set up departmental guidelines and protocols which restrict unnecessary $A \& E$ review of patients.

Nevertheless, the report of the national auditor and comptroller of A\&E services in England found that there was a national variation in the percentage of patients returning for review in A\&E clinics, ranging from 12 to $22 \%$. This suggests that there

Correspondence:

A.K. Maitra,

Consultant, Accident and Emergency Department, Royal Victoria Infirmary, Newcastle upon Tyne NE1 4LP, UK is scope for reduction in the number of patients reviewed in the A\&E clinics in a number of departments. A recommended target of $10-15 \%$ of total new A\&E attendances achieved nationally would eliminate up to 1.4 million patient-visits, freeing resources and time for other, especially new, attenders. ${ }^{1}$
In this urban teaching hospital $18-20 \%$ of a total of 50000 patients reattend for A\&E review. In order to reduce the number of clinic patients we needed to find out, in detail, why patients were being asked to return to the clinics and whether the reasons for referral were justified in accordance with departmental protocols and guidelines and clinical practice. The aim of this prospective study was to identify the reasons and appropriateness of referral to A\&E clinics by the A\&E doctors. Direct general practitioner (GP) referrals were excluded.

\section{PATIENTS AND METHODS}

The sample consisted of 500 patients seen in two periods of approximately 1 month during February and June 1993 (so as to include seasonal variation). The same group of doctors took part in the study during both periods.

A pro forma was devised listing likely reasons for clinic referral. All doctors were given standard departmental guidelines and protocols incorporating referral policy. Each doctor who wanted to refer a patient to the A\&E clinic after one initial visit completed the first part of the pro forma listing the age, sex, diagnosis (coded by region), clinical problem, referring doctor (coded by number) and the reason(s) for referral. On the return visit, the clinic doctor (registrar or more senior) compared the noted reason(s) for referral against the guidelines. If the reason(s) complied with the guidelines the visit was deemed to be appropriate, otherwise not. In the case of a small number of patients the noted reason(s) for referral did not match the guidelines, but the referring doctor was considered to have some reasonable concern. These cases were categorized as neither appropriate nor inappropriate (neutral response). The clinic doctor completed the final part of the form accordingly. No other criteria for assessment were used. 


\section{RESULTS}

\section{Demographic data}

Of the 500 patients studied 325 were males and 175 females; a male: female ratio of 2:1.

\section{Clinical details}

The nature of clinical conditions were as follows: (i) $236(47.2 \%)$ patients had soft tissue injuries, (ii) $164(32.8 \%)$ had fractures and dislocations, (iii) $57(11.4 \%)$ had lacerations, (iv) $25(5 \%)$ infections and (v) $17(3.4 \%)$ burns. The clinical details of one patient were missing.

The following anatomical sites were affected: (i) the hand (including digits) was most frequently involved (216:43.2\%) and more than half (117:54.2\%) of these patients had fractures/dislocations, (ii) the ankle or foot was the site of injury in $151(30.2 \%)$ patients, $40(26.5 \%)$ of whom had bone and joint injuries and (iii) the third most frequent site was the knee $(43: 8.5 \%)$.

\section{Appropriateness of referral}

The return visits of $395(79 \%)$ patients complied with the written guidelines and were judged to have been referred appropriately. In the case of $75(15 \%)$ the guidelines were not observed and these patients were deemed to have been inappropriately referred. Clinic doctors were unable to decide whether the referral was appropriate or not for $30(6 \%)$ patients for reasons described previously.

\section{Reasons for referral}

Figure 1 shows the different reasons for referral for all clinic patients. Further treatment of soft tissue injuries (149:28\%) and fractures and dislocations (137:27.4\%) was the most frequent reason for which the patients were brought to the clinic. A small number of patients was referred for dressings, delayed primary suture etc. Uncertainty about diagnosis and treatment (143:28.6\%) and worry about developing complications (sepsis, skin necrosis etc.) $(59: 11.8 \%)$ were cited as two other important reasons. The main reasons for referral i.e. need for further treatment and uncertainty about diagnosis and treatment were also the common reasons for appropriate referral. By far the highest percentage of inappropriate referral $(61.5 \%)$ was the group where no specific reason on the proforma was cited. The percentage of appropriate and inappropriate referral at each anatomical site identified the forearm/ wrist and ankle/foot as sites that are frequently involved in those who were inappropriately referred. The numbers of patients with abdominal and hip/ pelvis injuries who were referred inappropriately were too small to be significant.

\section{Doctor variation}

The percentage of referral was similar amongst the A\&E doctors except one.

\section{DISCUSSION}

The National Audit report ${ }^{1}$ highlighted the need for efficient use of available resources in A\&E departments. One way to achieve this objective would be to reduce the number of return patients. This laudable aim can be achieved in two ways which are not mutually exclusive: review and change existing clinical practice and increase compliance with the guidelines by all doctors, especially the trainees, through education. Our study has shown

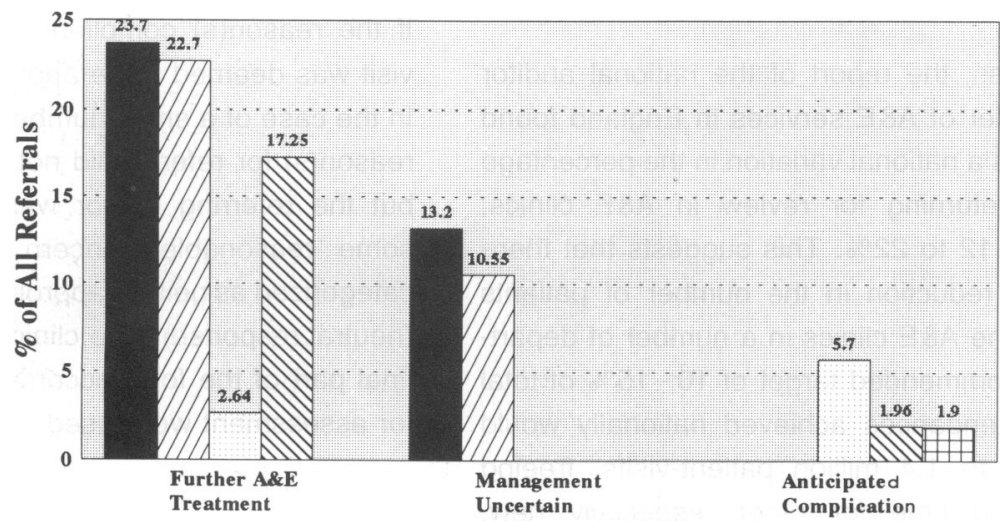

Reason For referral
Fig. 1. Distribution of the reasons for referral to clinics. $\square$, Soft tissue;

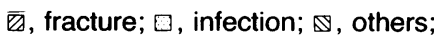
and skin necrosis. 
that the scope for reduction of referrals by education and increasing compliance with the guidelines is small, accounting for about one-fifth of the total number reviewed. Even if this were achieved one must be aware of the danger that rigorous enforcement of guidelines could lead to undue pressure on young doctors with deleterious results for patient care.

In order to reduce the number of clinic patients significantly, a change of clinical practice would be necessary, namely, drastically curtailing the definitive and follow-up treatment of a range of fractures/dislocations and soft tissue injuries. Before we go down this road, we need to consider: (1) the merits of the existing policy of patient management (i.e. patient welfare), (2) training of career grade staff and (3) job-satisfaction of the permanent staff. Soft tissue injuries vary in their degree of severity and it is recognized that moderate to severe injuries require supervised treatment in which A\&E departments have developed expertise over a number of years. These patients would not be treated in specialized orthopaedic or plastic surgery units, which are already overstretched with their particular clinical problems. GPs, in general, lack the appropriate training and resources to manage these patients. Patients prematurely discharged or undertreated are likely to represent in an unscheduled way. More resources from all areas would then have to be used in an effort to treat the ensuing avoidable morbidity, delaying patient recovery and risking patients' complaints and possible litigation. With regard to the follow-up management of fractures/dislocations (the second largest group in our study, $27.4 \%$ ), there is a great deal of variation in practice in the country. However, a significant number of A\&E departments have developed expertise and demonstrated good results in treating hand injuries including fractures/ dislocations. ${ }^{2}$ One recent study ${ }^{3}$ demonstrated that proximal phalangeal fractures of the hand can be very successfully managed by $A \& E$ doctors using the correct guidelines to refer complicated injuries to the specialists. This policy results in patients receiving quick and satisfactory treatment and, at the same time, reducing the workload of specialist departments. The continuing management of these groups of patients (and other patient groups for which a special interest and expertise has been developed in A\&E departments) allows training of doctors in the further care of the patients under the supervision of senior A\&E doctors and provides avenues for clinical research and audit. Last but not least, it continues to set challenges and gives a sense of satisfaction to the ever-increasing number of senior staff being appointed to this specialty.

In conclusion, this study demonstrates that there is scope for a small to moderate reduction (15-20\%) in the number of $A \& E$ clinic patients by ensuring more effective compliance with guidelines. This can be achieved by the following measures. (1) Dissemination: adequate imparting of guidelines to the staff from the onset of their appointment. (2) Education: education and training in a seminar setting concentrating on particular guidelines, explaining the rationale underlying the guidelines and the potential benefit from their usage. (3) Reminders: both patient specific and general non-specific reminders. (4) Peer support: promotion and endorsement by peers of guidelines. (5) Audit and feedback. (6) Evaluation: to assess whether improved quality of patient care follows compliance of guidelines. (7) Updating: modification of guidelines to reflect change in knowledge and local circumstances. However, the specialty of A\&E must not sacrifice its identity by doing away with its own clinical practice but should allow local and regional variation within an accepted limit.

\section{ACKNOWLEDGEMENT}

We acknowledge the assistance of Mrs J. Lally of the Audit Office for statistical analysis and preparation of illustrations.

\section{REFERENCES}

1. National Audit Office Report By the Comptroller and Auditor General (1992) NHS Accident \& Emergency Departments in England, pp. 18-20. HMSO, London.

2. Phair I.C., Quinton D.N. \& Allen M.J. (1989) The conservative management of volar avulsion fractures of the P.I.P. joint. The Journal of Hand Surgery 14B(2), 168-170.

3. Maitra A.K. \& Burdett-Smith P. (1992) The conservative management of proximal phalangeal fractures of the hand in an accident and emergency department. The Journal of Hand Surgery 17B(3), 322-336. 